\title{
Operative management of ascending aorta thrombus and bilateral pulmonary embolism in the setting of breast cancer
}

\author{
Jorge Alcocer ${ }^{1}$, María Ascaso $^{2}$, Eduard Quintana $^{2}$, Elena Sandoval ${ }^{3}$, and Manuel Castella ${ }^{1}$ \\ ${ }^{1}$ Department of Cardiovascular Surgery, Institut Clinic Cardiovascular, Hospital Clínic de \\ Barcelona, University of Barcelona, Barcelona, Spain \\ ${ }^{2}$ Hospital Clínic \\ ${ }^{3}$ Hospital Clínic, Cardiovascular Surgery
}

November 24, 2020

\begin{abstract}
Pulmonary embolism and concomitant floating aortic thrombus are a rare and potentially life-threatening association. Several therapeutic options are available and best management can be controversial when these conditions coexist. We describe a case of a 79-year-old woman presented with massive pulmonary embolism and simultaneous floating thrombus in the ascending aorta. She underwent concomitant ascending aortic replacement and surgical pulmonary embolectomy with an uneventful postoperative recovery. Open surgical repair is a one stage repair approach that may offer the most efficient treatment to allow survival.
\end{abstract}

Operative management of ascending aorta thrombus and bilateral pulmonary embolism in the setting of breast cancer

Aortic thrombus and pulmonary embolism

Jorge Alcocer MEBCTS*, María Ascaso MD, Eduard Quintana PhD, Elena Sandoval FEBCTS, Manuel Castellá PhD

Cardiovascular Surgery Department, Hospital Clinic, Barcelona, Spain

${ }^{*}$ Corresponding author:

Dr. Jorge Alcocer

Department of Cardiovascular Surgery, Hospital Clinic Barcelona

C/ Villarroel 170, 08036, Barcelona, Spain

Phone: + 34932275400 (Ext: 387006)

E-mail: alcocer@clinic.cat

Funding: There has not been any source of funding for this work.

Disclosures: The authors have no relationships relevant to the contents of this paper to disclose.

Institutional Review Board approval: N/A

Clinical trial registration: N/A 
Consent statement: Oral consent was obtained and the patient voluntarily authorized us to write a case report about her treatment and to share medical information related to it (diagnostic images, photos and videos)

Data availability statement: Data sharing is not applicable to this article as no datasets were generated during the current study.

\section{Abstract}

Pulmonary embolism and concomitant floating aortic thrombus are a rare and potentially life-threatening association. Several therapeutic options are available and best management can be controversial when these conditions coexist.

We describe a case of a 79-year-old woman presented with massive pulmonary embolism and simultaneous floating thrombus in the ascending aorta. She underwent concomitant ascending aortic replacement and surgical pulmonary embolectomy with an uneventful postoperative recovery.

Open surgical repair is a one stage approach that may offer the most efficient treatment to allow survival.

\section{Key words}

Pulmonary embolism, aortic thrombus, breast cancer, pulmonary embolectomy, aortic replacement, hypercoagulability

\section{Introduction}

Pulmonary embolism (PE) is a medical condition usually managed with anticoagulation or thrombolysis, however in certain cases surgical management can be considered as the best therapeutic intervention ${ }^{1}$. Floating aortic thrombus can lead to catastrophic systemic embolism and operative management prevents this complication $^{2,3}$. Several reports of successful systemic anticoagulation alone have been reported. Concomitant presentation of both conditions poses extra challenge to clinical decision making to ensure the best possible outcome.

\section{Case}

A 79-year-ol woman presented at emergency department complaining of progressive dyspnea, cough with mild hemoptysis and pleuritic chest pain for one week. The patient's medical history was remarkable for the presence of systemic arterial hypertension and recent surgery for stage I breast cancer three months ago. After surgery, the patient started adjuvant therapy with aromatase inhibitor letrozole and local radiotherapy until the time of admission. She disclosed neither smoking habit nor alcohol consumption. Systemic temperature was $37^{\circ} \mathrm{C}$, her oxygen saturation was $95 \%$ with room air and systemic blood pressure was 120/60 mmHg. Physical examination revealed mild tachypnea and a heart rate of 110 beats per minute. Cardiac and pulmonary auscultation did not show any other abnormality. Peripheral pulses were palpable in all extremities and no swelling was present in lower limbs.

Twelve-lead surface electrocardiogram and chest-x-ray did not show any pathological finding. Laboratory testing revealed a normal blood cell count, renal and liver function and lipid profile. Cardiac markers were slightly elevated (high-sensivity troponin I $106 \mathrm{ng} / \mathrm{L}$, normal range $<45 \mathrm{ng} / \mathrm{L}$ ) and D-dimer was $1200 \mathrm{ng} / \mathrm{ml}$ (normal range $<250 \mathrm{ng} / \mathrm{ml}$ ). Basic clotting test showed normal platelet count, international normalized ratio, activated partial thromboplastin time and fibrinogen levels. Transthoracic echocardiography showed normal left ventricular function and dilated right ventricle with preserved contractility. There were no valvular abnormalities, shunts or pericardial effusion and visualized ascending aorta was normal. Finally, computed tomography (CT) pulmonary angiogram showed a saddle PE extending into the lobar arteries of both lungs (Figure 1A). Furthermore, there was an intraluminal filling defect in distal ascending aorta corresponding to a floating thrombus (Figure 1B).

The patient was admitted at the ICU due to progressive hypoxemia and intravenous heparin was initiated. Diagnosis of simultaneous high-risk PE and a floating thrombus in the ascending aorta, as a potential source 
of stroke or peripheral emboli, mandated an open surgical repair. In order to rule out metastatic disease, a total body CT scan and bone scintigraphy were performed and did not reveal any lesion suggestive of cancer metastasis.

After median sternotomy, right axillary artery and bicaval cannulation, cardiopulmonary bypass was instituted and cardioplegic arrest was obtained by means of retrograde cold blood cardioplegia. The patient underwent thrombus resection and ascending aortic replacement with a $28 \mathrm{~mm}$ dacron graft. Due to distal location of thrombus and calcification of the distal portion of the ascending aorta, an open distal anastomosis was performed with systemic circulatory arrest, moderate hypothermia $\left(25^{\circ} \mathrm{C}\right)$ and bilateral selective cerebral perfusion. After systemic and coronary reperfusion, we carried out a surgical pulmonary thromboembolectomy through a separate longitudinal arteriotomy in left and right pulmonary arteries (Supplementary video)

Aortic thrombus was $2 \mathrm{~cm}$ in size, pale and pedunculated over a calcified atheromatous plaque in the anterior wall of the distal ascending aorta (Figure 2). Moreover, a great amount of soft and dark red thrombus was taken out from both pulmonary arteries extending into lobar and segmental arteries (Figure 3).

The postoperative course was uneventful and a thorough coagulation testing was performed postoperatively to rule out a hypercoagulable state. Therefore, anticardiolipine antibodies, lupus anticoagulant, B2glycoprotein, protein C activity, antithrombin activity and homocysteine levels were strictly normal. Postoperative CT angiogram revealed a normal appearance of ascending aortic graft and good perfusion of both pulmonary arteries with no residual pulmonary emboli (Figure 4).

Finally, the patient was discharged at postoperative day 10, aromatase inhibitor letrozole was discontinued and direct oral anticoagulant edoxaban was initiated. At 6 months, the patient is completely recovered with good functional class.

\section{Discussion}

In this case, we found a very unusual association of two thrombotic conditions with different pathogenic mechanisms and potential life-threatening complications. Recent history of cancer and current treatment with letrozole were considered the only factors associated with this prothrombotic state ${ }^{4}$.

On the one hand, floating aortic thrombus is an unusual disease, frequently detected in the setting of diagnostic work-up after stroke or peripheral embolism. Thrombi are mostly located in the aortic arch and can be attached either on aortic plaques or visually normal wall ${ }^{5}$. The pathophysiology of these lesions is unclear, since thrombophilic states are not frequently found ${ }^{2}$, as in this case. Medical management with heparinization, endovascular stenting and surgery have been proposed and previously published, but comparative data are not available. ${ }^{5,6}$

On the other hand, usual treatment of PE consists in systemic anticoagulation. Systemic fibrinolysis is indicated in case of hemodynamic or cardiac compromise and surgical embolectomy is reserved for those cases where fibrinolysis is contraindicated or failed. ${ }^{7}$ This case was a high-risk PE without hemodynamic instability and could only be managed with anticoagulation alone and systemic fibrinolysis in case of hemodynamic deterioration. ${ }^{1}$ However, the presence of floating thrombus in the ascending aorta with a high risk of stroke led to concomitant surgical repair of both conditions. Some circumstances other than contraindication for fibrinolysis can make surgical pulmonary embolectomy the best approach PE management.

\section{Authors Contribution}

Jorge Alcocer provided concept and design.

María Ascaso drafted the manuscript

Eduard Quintana, Elena Sandoval and Manuel Castellá provided critical revision and approved the submitted version

\section{References}


1. Konstantinides SV, Meyer G, Becattini C, et al. 2019 ESC Guidelines for the diagnosis and management of acute pulmonary embolism developed in collaboration with the European Respiratory Society (ERS). Eur Respir J . 2019;54(3):1901647

2. Erbel R, Aboyans V, Boileau C, et al. 2014 ESC Guidelines on the diagnosis and treatment of aortic diseases: Document covering acute and chronic aortic diseases of the thoracic and abdominal aorta of the adult. The Task Force for the Diagnosis and Treatment of Aortic Diseases of the European Society of Cardiology. Eur Heart J . 2014;35(41):2873-2926.

3. Laperche T, Laurian C, Roudaut R, Steg PG. Mobile thromboses of the aortic arch without aortic debris. A transesophageal echocardiographic finding associated with unexplained arterial embolism. The Filiale Echocardiographie de la Societe Francaise de Cardiologie. Circulation . 1997;96(1):288-294.

4. Bundred NJ. The effects of aromatase inhibitors on lipids and thrombosis. Br J Cancer . 2005;93 Suppl 1:S23-S27.

5. Toyama M, Nakayama M, Hasegawa M, Yuasa T, Sato B, Ohno O. Direct oral anticoagulant therapy as an alternative to surgery for the treatment of a patient with a floating thrombus in the ascending aorta and pulmonary embolism. J Vasc Surg Cases Innov Tech . 2018;4(2):170-172.

6. Goette A, Hammwohner M, Dierkes J, et al. Aortic thrombus and pulmonary embolism in a patient with hyperhomocysteinemia. Nat Clin Pract Cardiovasc Med . 2006;3(7):396-399.

7. Carvalho EM, Macedo FIB, Panos AL, et al. Pulmonary embolectomy: recommendation for early surgical intervention. J Card Surg. 2010;25(3):261-266.

Figure 1

A. Preoperative CT pulmonary angiogram shows a saddle pulmonary embolism (Red asterisk)

B. Chest CT reveals a floating thrombus in the anterior wall of distal ascending aorta (black asterisk) and pulmonary embolism extending into lobar arteries (red asterisk)

Figure 2

Intraoperative view of thrombus attached to an atheromatous plaque in the ascending aortic wall

Figure 3

Surgical pulmonary embolectomy specimen from right (A) and left side (B)

Figure 4

A. Postoperative CT angiogram showing normal appearance of ascending aortic graft

B. Postoperative pulmonary angio-CT showing no residual pulmonary emboli and normal perfusion of main pulmonary arteries and lobar branches

Hosted file

Imagenes case report.pptx available at https://authorea.com/users/378402/articles/494919operative-management-of-ascending-aorta-thrombus-and-bilateral-pulmonary-embolism-inthe-setting-of-breast-cancer 